\title{
Identification of Candidate Genes of Male Sexual Development From Androgenic Gland in Macrobrachium Nipponense Through Performing Long-reads and Next Generation Transcriptome Sequencing After Eyestalk Ablation
}

\section{Shubo Jin}

Key Laboratory of Freshwater Fisheries and Germplasm Resources Utilization, Ministry of Agriculture, Freshwater Fisheries Research Center, Chinese Academy of Fishery Sciences

\section{Yuning Hu}

Key Laboratory of Freshwater Fisheries and Germplasm Resources Utilization, Ministry of Agriculture, Freshwater Fisheries Research Center, Chinese Academy of Fishery Sciences

Hongtuo Fu ( $\nabla$ fuht@ffrc.cn )

Key Laboratory of Freshwater Fisheries and Germplasm Resources Utilization, Ministry of Agriculture, Freshwater Fisheries Research Center, Chinese Academy of Fishery Sciences

\section{Sufei Jiang}

Key Laboratory of Freshwater Fisheries and Germplasm Resources Utilization, Ministry of Agriculture, Freshwater Fisheries Research Center, Chinese Academy of Fishery Sciences

\section{Yiwei Xiong}

Key Laboratory of Freshwater Fisheries and Germplasm Resources Utilization, Ministry of Agriculture, Freshwater Fisheries Research Center, Chinese Academy of Fishery Sciences

\section{Hui Qiao}

Key Laboratory of Freshwater Fisheries and Germplasm Resources Utilization, Ministry of Agriculture, Freshwater Fisheries Research Center, Chinese Academy of Fishery Sciences

\section{Wenyi Zhang}

Key Laboratory of Freshwater Fisheries and Germplasm Resources Utilization, Ministry of Agriculture, Freshwater Fisheries Research Center, Chinese Academy of Fishery Sciences

\section{Yongsheng Gong}

Key Laboratory of Freshwater Fisheries and Germplasm Resources Utilization, Ministry of Agriculture, Freshwater Fisheries Research Center, Chinese Academy of Fishery Sciences

\section{Yan Wu}

Key Laboratory of Freshwater Fisheries and Germplasm Resources Utilization, Ministry of Agriculture, Freshwater Fisheries Research Center, Chinese Academy of Fishery Sciences

Yin Fu 
Key Laboratory of Marine and Estuarine Fisheries, Ministry of Agriculture, East China Sea Fisheries Research Institute, Chinese Academy of Fishery Sciences, Shanghai 200090, P. R. China

\section{Research Article}

Keywords: Macrobrachium nipponense, Long-reads transcriptome, Eyestalk ablation, Androgenic gland, Male sexual development

Posted Date: March 2nd, 2021

DOl: https://doi.org/10.21203/rs.3.rs-244791/v1

License: (9) This work is licensed under a Creative Commons Attribution 4.0 International License. Read Full License 
1 Identification of candidate genes of male sexual development from androgenic gland in

2 Macrobrachium nipponense through performing long-reads and next generation transcriptome

3

4 $5 \quad$ Wenyi Zhang ${ }^{1}$, Yongsheng Gong ${ }^{1}$, Yan $\mathrm{Wu}^{1}$

6

sequencing after eyestalk ablation Sciences, Wuxi 214081, P. R. China R. China *Corresponding author: Hongtuo Fu E-mail address: fuht@,ffrc.cn

Tel.: +86.51085558835

\section{Abstract}

Shubo $\mathrm{Jin}^{1}$, Yin $\mathrm{Fu}^{2}$, Yuning $\mathrm{Hu}^{3}$, Hongtuo $\mathrm{Fu}^{1,3 *}$, Sufei Jiang ${ }^{1}$, Yiwei Xiong ${ }^{1}$, Hui Qiao ${ }^{1}$,

1. Key Laboratory of Freshwater Fisheries and Germplasm Resources Utilization, Ministry of Agriculture, Freshwater Fisheries Research Center, Chinese Academy of Fishery

2. Key Laboratory of Marine and Estuarine Fisheries, Ministry of Agriculture, East China Sea Fisheries Research Institute, Chinese Academy of Fishery Sciences, Shanghai 200090, P.

3. Wuxi Fisheries College, Nanjing Agricultural University, Wuxi 214081, China Freshwater Fisheries Research Center, Chinese Academy of Fishery Sciences

9 Shanshui East Road, Wuxi 214081, Jiangsu Province, P. R. China

The eyestalk of crustacean species contained many neurosecretory structures, affected the process of reproduction, molting, metabolism of glucose and other function in crustaceans. In this study, we aimed to selected important metabolic pathways and candidate genes involved in the male sexual development through performing the long-reads and next generation transcriptome sequencing of androgenic gland after the ablation of eyestalk from Macrobrachium nipponense. qPCR analysis revealed that the mRNA expression of Mn-IAG was significantly increased after ablation both of the single-side (SS) and double-side (DS) 
eyestalk, compared with that of control group (CG). The long-reads transcriptome generated 49,480 non-redundant transcripts. A total of 1,319, 2,092 and 4,351 differentially expressed genes (DEGs) were identified between CG vs SS, SS vs DS and CG vs DS, respectively, indicating the ablation of double-side eyestalk has more important regulatory roles than that of single-side ablation on male sexual development, which was consistent with that of qPCR analysis. Cell cycle, Cellular senescence, Oxidative phosphorylation, Glycolysis/Gluconeogenesis and Steroid hormone biosynthesis were the main enriched metabolic pathways in all of these three comparisons, and the important genes from these metabolic pathways were also selected. The qPCR verifications of 10 GEDs were as the same as that of RNA-seq. The qPCR and RNAi analysis of Hydroxysteroid dehydrogenase like 1 (HSDL1) revealed that HSDL1 has the positive regulatory effected on testis development. This study provided valuable evidences on male sexual development in $M$. nipponense, promoting the studies on male sexual development in other crustacean as well.

Key words: Macrobrachium nipponense; Long-reads transcriptome; Eyestalk ablation; Androgenic gland; Male sexual development

\section{Introduction}

The oriental river prawn, Macrobrachium nipponense (Crustacea; Decapoda; Palaemonidae), is widely distributed in China and other Asian countries [1-3], which is an important commercial species with the annual aquaculture production reached of 205,010 tons in 2016 [4]. As the same as other Macrobrachium species, male prawns grow faster and reach larger size at the harvest time [2]. Thus, male prawns are preferred in the M. nipponense aquaculture. In addition, the rapid development of testis in the reproductive season is another main problem, restricted the sustainable development of $M$. nipponense. The previous studies revealed that the testis of a new born M. nipponense can reach sexual maturity within 40 days after hatching [5]. Thus, inbreeding will be happened between the new born prawns. Inbreeding will lead to 
the decrease of the ability of resistance to adversity in their offspring, the small scale of market prawn, and the degradation of germplasm resources. Therefore, it is urgently needed to fully understand the male sexual differentiation and development mechanism, with the aims of establishment of the technique to produce all male progeny on a commercial scale, and to regulate the process of testis development in M. nipponense.

Androgenic gland is a special tissue in crustacean species. It has been proven to play essential roles in male sexual differentiation and development in crustacean species. Many studies reported that the androgenic gland and its secreted hormones promote the driving of male sexual differentiation, the establishment of male sexual characteristics, and the development of the testes in crustacean species [6-7]. The ablation of androgenic gland from male Macrobrachium rosenbergii resulted in the sex reversal to "neo-female". Insulin-like androgenic gland hormone (IAG) is an important hormone, secreted by androgenic gland. IAG was proven to promote the male sexual differentiation and development in many crustacean species [8-10]. The knockdown of IAG expression by RNAi in male M. rosenbergii can also result in the sex reversal [11]. Based on the importance of androgenic gland in male sexual differentiation and development in crustacean species, the studies on androgenic gland have been became the hot topic in recent years. A series of transcriptomes of androgenic gland have been constructed in M. nipponense [12-14], and a series of important genes from androgenic gland have been showed to play essential roles in male sexual development [15-18]. In addition, the histological observations during different post-larval developmental stages indicated that the development of androgenic gland has regulatory roles on the development of testis [5].

The eyestalk of crustacean species has many neurosecretory structures. The X-organ-SG complex (XO-SG) was identified as a principal neuroendocrine gland located in the eyestalk in crustaceans [19]. It stores and releases the crustacean hyperglycemic hormone $(\mathrm{CHH})$ superfamily neurohormones, including $\mathrm{CHH}$, iontransport peptides (ITP), gonad-inhibiting 
hormone $(\mathrm{GIH})$, molt inhibiting hormone $(\mathrm{MIH})$, and mandibular organ-inhibiting hormone $(\mathrm{MOIH})$, playing essential roles in reproduction [20-22], molting [23-25], metabolism of glucose [26-27] and other function [28-30]. Knockdown the expression of GIH by RNAi promote the ovarian development in M. nipponense [31]. Knockdown the expression of MIH by RNAi promote the molting in M. nipponense [32]. CHH has been proven to promote testis development in M. nipponense [33].

In this study, we aimed to select the vital metabolic pathways and genes involved in the male sexual differentiation and development in $M$. nipponense through performing the longreads and next generation transcriptome profiling analysis of androgenic gland after the ablation of single-side and double-side eyestalk. The functions of Hydroxysteroid dehydrogenase like 1 (HSDL1) were further analyzed in depth by using qPCR analysis and RNAi. This study provided valuable evidences on the studies of male sexual differentiation and development in M. nipponense, as well as other crustacean species.

\section{Results}

\section{The expression analysis of Mn-IAG after eyestalk ablation}

The mRNA expression of Mn-IAG were measured in three groups, including CP, SS and DS (Figure 1). The mRNA expression of Mn-IAG were increased with time of eyestalk ablation in SS group and DS group. The mRNA expressions of Mn-IAG were about 5-folder higher at day 4 and day 7 than that of day 1 in both SS and DS group $(\mathrm{P}<0.01)$. However, the Mn-IAG expression was only slightly higher at day 7 than that of day 4 in both SS and DS group, and showed no significant difference $(\mathrm{P}>0.05)$. The Mn-IAG expression in the DS group was almost 2-folder higher than SS group at the same day, and showed significant difference $(\mathrm{P}<$ $0.05)$

\section{Long-read transcriptome}


A total of $22.83 \mathrm{~GB}$ clean data was generated in the long-reads transcriptome. A total of 160,496 high-quality transcripts were obtained with a mean length of 2,230 bp. Finally, 49,480 nonredundant transcripts were identified in the long-reads transcriptome. All of the non-redundant transcripts were compared with the non-redundant protein database and nucleotide sequences in NCBI, in order to identify their putative functions. A total of 37,355(74.94\%) unigenes were annotated in $\mathrm{Nr}$ database. The other unannotated transcripts represent novel genes whose functions need further investigation.

GO and COG analysis aimed to provide a structured vocabulary to describe gene products. A total of 19,673 (39.76\%) unigenes were assigned to GO database, comprised of 52 functional groups (Figure 2). The number of unigenes in each functional group ranged from 1 to 10,057. A total of 13,395 (27.07\%) unigenes were highly matched with the known proteins in COG database, classified into 25 functional groups (Figure 3). The number of unigenes in each functional group ranged from 1 to 6,793 . KEGG analysis aimed to revealed the regulatory relationship between the unigenes in the long-reads transcriptome. A total of 18,618 $(36.72 \%)$ unigenes were highly matched the known genes in KEGG databse, mapped onto 264 metabolic pathways.

\section{Identification of differentially expressed genes}

The differentially expressed genes were identified, using the criterion of $>2.0$ as up-regulatory genes and $<0.5$ as down-regulatory genes, and P-value $<0.05$. A total of 1,319 differentially expressed genes (DEGs) were identified between CG and SS, including 713 up-regulated genes and 606 down-regulated genes. A total of 2,092 DEGs were identified between SS and DS, including 1,036 up-regulated genes and 1,056 down-regulated genes. A total of 4,351 DEGs were found between CG and DS, including 2163 up-regulatory genes and 2188 downregulatory genes. KEGG analysis revealed that Cell cycle, Cellular senescence, Oxidative 
phosphorylation, Glycolysis/Gluconeogenesis and Steroid hormone biosynthesis were the main enriched metabolic pathways in all of these three comparisons.

A total of 15 DEGs were selected from these enriched metabolic pathways, which were listed in Table 3. These genes were differentially expressed in at least two of these three comparisons. Cyclin B3, MAD2A, Polo-like kinase 1, Cyclin A, Cdc2 kinase, and Cyclin B were mainly found in the metabolic pathways of Cell cycle and Cellular senescence, which were differentially expressed in all of these three comparisons. SDHB, Cytochrome c oxidase assembly protein COX11 and Cytochrome c oxidase subunit 7A1 were mainly selected from the metabolic pathways of oxidative phosphorylation. Acetyl-coenzyme A synthetase 2-like, Fructose-bisphosphate aldolase, and Alcohol dehydrogenase class -3 were mainly differentially expressed in the metabolic pathways of Glycolysis/Gluconeogenesis. Estrogen sulfotransferase, 3 beta-hydroxysteroid dehydrogenase and HSDL1 were identified from the metabolic pathways of steroid hormone biosynthesis.

\section{qPCR verification}

qPCR analysis was used to verify the expressions of important DEGs in the androgenic gland from the CG, SS and DS prawns. The qPCR analysis showed the same expression pattern with that of RNA-seq (Figure 4). Six DEGs from the metabolic pathways of Cell cycle and Cellular senescence showed the lowest expressions in the CG prawns, and highest expressions in the DS prawns, including Cyclin B3, MAD2A, Polo-like kinase 1, Cyclin A, Cdc2 kinase, and Cyclin B. The mRNA expressions of these DEGs in the DS prawns showed significant difference with that of CG prawns and SS prawns $(p<0.01)$. The mRNA expressions of estrogen sulfotransferase, and alcohol dehydrogenase class -3 showed no significant difference between the CG prawns and the SS prawns $(p>0.05)$, whereas showed significant difference with that of DS prawns $(p<0.05)$. The mRNA expression of SDHB and HSDL1 between the 
prawns of SS and DS showed no significant difference $(p>0.05)$, whereas showed significant difference with that of CG prawns $(p<0.05)$.

\section{Expression analysis of Mn-HSDL1}

The previous study has been reported that the Mn-HSDL1 mRNA showed the highest expression level in the hepatopancreas, followed by the testis, which showed significant difference with other tested tissues $(p<0.05)$. The expression in the hepatopancreas and testis was 187 and 90-folder higher than that in the brain [48]. The mRNA expressions of Mn-HSDL1 in different developmental stages were measured by using qPCR (Figure 5). The Mn-HSDL1 expression in the larval developmental stages were generally higher than that of post-larvaldevelopmental. The highest expression level was observed in larval day 5 (L5), while it showed no significant difference with other tested stage $(p>0.05)$. During the post-larval developmental stages, the lowest expression level was observed in post-larval day 5 (PL5), and then gradually increased. The highest expression level was observed in PL25 $\widehat{\jmath}$, which was 3,72 and 1.94-folder higher than that of PL5 and PL25 9 , respectively.

\section{RNAi analysis of Mn-HSDL1}

RNAi was used to analyze the functions of Mn-HSDL1 on male sexual development in $M$. nipponense. qPCR analysis revealed that the expression of Mn-HSDL1 remained stable in the control group after the injection of Mn-HSDL1 dsRNA $(\mathrm{P}>0.05)$. However, the expression of Mn-HSDL1 significantly deceased at day 7 and 14 after the injection of Mn-HSDL1 dsRNA. The decrease reached to $96 \%$ and $90 \%$ at day 7 and 14, respectively, compared with that in control group (Figure 6-A).

The expressions of Mn-IAG were also measured in the cDNA template from the same prawns (Figure 6-B). According to the qPCR analysis, the expression of Mn-IAG at day 1 in control group was slightly higher than that of day 7 and day 14, while it generally remained stable. In RNAi group, the expressions of Mn-IAG were significantly decreased at day 7 and 
day 14 after the injection of Mn-HSDL1 dsRNA. The expression decreased about $61 \%$ and 54\% at day 7 and 14, respectively, compared with that in control group.

\section{Histological observations of testis after RNAi}

According to the histological observations, sperms were the dominant cell type in the testis from control group, and only a limited number of spermatogonias and spermatocytes were observed. In RNAi group, the number of sperms were gradually deceased with the time of MnHSDL1 dsRNA treatment, and sperms were rarely found at day 14 after Mn-HSDL1 dsRNA treatment. However, the number of spermatogonias were increased (Figure 7).

\section{Discussion}

The eyestalk of crustaceans secreted many neurosecretory structures, mediated the reproduction, molting and metabolism of glucose in crustaceans [19-30]. The important neurosecretory structures include $\mathrm{CHH}, \mathrm{ITP}, \mathrm{GIH}, \mathrm{MIH}$ and MOIH. Previous study reported that RNAi was used to knockdown the expression of GIH in female M. nipponense, promoting the ovarian developmental process [31]. This study aimed to analyze the effects of eyestalk on male sexual development. qPCR analysis revealed that the mRNA expression of Mn-IAG significantly increased at day 4 and day 7 after eyestalk ablation in both single-side and doubleside ablation, compared with that of day 1 , and the expression in double-side ablation was significantly higher than that of single-side ablation and normal prawn at the same day, which was consistent with the previous studies [34-36]. However, the expression between day 4 and day 7 showed no significant difference in both single-side and double-side ablation. IAG has been reported to promote the male sexual differentiation and development in crustaceans [810]. Thus, the increase of Mn-IAG expression after the ablation of eyestalk indicated that eyestalk has negative effects on male sexual differentiation and development in M. nipponense, which has the similar mediated functions on ovarian development in M. nipponense.

To the best of our knowledge, this is the first long-reads transcriptome in M. nipponense. 
The combination of long-reads and next generation transcriptome sequencing can obtain transcripts with better integrity and quality for further gene structure and function analysis. The accuracy and length of the transcripts of the long-reads transcriptome are further improved and optimized through correcting by the next generation transcripts. Thus, it is a suitable strategy for the species without reference genome. The genes related to the male sexual development were predicted to be mainly found in the functional groups of Cell, Cell part, Cellular process and Binding in the GO assignment, and in the functional groups of General function prediction only, Signal transduction mechanisms and Posttranslational modification, protein turnover, chaperones in the COG classification, which were consistent with the previous studies [37-38]. The gene sequences from this long-reads transcriptome provide valuable information for the analysis of gene structure and gene function.

The number of DEGs between CG vs DS were 4,351, which were significantly more than the number of DEGs between CG vs SS and SS vs DS, indicating the ablation of double-side eyestalk has more regulatory roles on male sexual development in M. nipponense, which was consistent with the qPCR analysis. KEGG analysis revealed that Cell cycle, Cellular senescence, Oxidative phosphorylation, Glycolysis/Gluconeogenesis and Steroid hormone biosynthesis were the main enriched metabolic pathways in all of these three comparisons. Previous studies have been predicted the important roles of Oxidative phosphorylation, Glycolysis/Gluconeogenesis and Steroid hormone biosynthesis in the mechanism of male sexual development in $M$. nipponense [37-38], predicting the DEGs from these metabolic pathways in this study may play essential roles in male sexual development in $M$. nipponense.

The male sexual development will be vigorous after ablation the eyestalks. The transcriptome profiling analysis revealed that cell cycle and cell senescence were the most enriched metabolic pathway in all of these three comparisons. The cell cycle is a ubiquitous and complex process to ensure the correct during cell proliferation, in order to prevent the 
copies of DNA damage, genetic derangement and other errors. Cyclins and cyclin-dependent kinase play essentials roles in this process [39-40]. Cellular senescence is defined as irreversible cell cycle arrest caused by different forms of stress. These stresses include telomere shortening, other forms of genotoxic stress, or mitogens or inflammatory cytokines, catalyzing the activation of the p53 tumor suppressor and/or the cyclin-dependent kinase inhibitor p16 [41-42]. The dramatic enrichment of DEGs in these two metabolic pathways indicated that cell cycle and cell senescence play essential roles in the proofreading process when cells make copies of themselves. Four DEGs were enriched in both of the cell cycle and cell senescence, including cyclin A, cyclin B, cyclinB3 and cyclin-dependent kinase 2 (cdk2). Cyclin A is a vital component of the cell-cycle machinery, which can activate two different cyclin-dependent kinases (Cdk1 and Cdk2), functioning in both S-phase and mitosis [43-45]. Cdk1/cyclin B, which is also known as maturation promoting factor (MPF), is one of the main protein kinases. It activates and serves as master regulator for the M-phase transition, phosphorylating and activating other downstream protein kinases, and directly phosphorylating several structural proteins involved in cellular reorganization [46-48]. Cdk family includes $8 \mathrm{cdk}$ genes which can combine with different types of cyclins to form complexes, regulating the process of cell transition from $\mathrm{G} 1$ phase to $\mathrm{S}$ phase or $\mathrm{G} 2$ phase to $\mathrm{M}$ phase and exit from $\mathrm{M}$ phase. $\mathrm{Cdk} 2$ is a member of a highly conserved family of protein kinases, regulating the eukaryotic cell cycle [49-51].

ATP is an unstable high-energy compound that is the most direct energy source in organisms. It is widely acknowledged that ATP is essential for the activities in an orgamisn, including male differentiation and development. In the present study, oxidative phosphorylation and glycolysis/gluconeogenesis were the main enriched metabolic pathways in all of the three comparisons. Oxidative phosphorylation occurs in the inner membrane of mitochondria of eukaryotic cells or in the cytoplasm of prokaryotes. The energy released from 
the oxidation of substances in vivo promote the coupling reaction between ADP and inorganic phosphate to synthesize ATP through the respiratory chain [52]. Glycolysis/gluconeogenesis promote the conversion of glucose $\left(\mathrm{C}_{6} \mathrm{H}_{12} \mathrm{O}_{6}\right)$ into pyruvate $\left(\mathrm{CH}_{3} \mathrm{COCOO}-+\mathrm{H}+\right)$, releasing free energy to form the high energy molecules ATP and reduced nicotinamide adenine dinucleotide [53]. Three DEGs were respectively selected from both of the metabolic pathways of oxidative phosphorylation and glycolysis/gluconeogenesis. SDHB is a DEG, which was down-regulated between CG vs SS and CG vs DS. SDHB was also predicted to be involved in the mechanism of male sexual development in M. nipponense. SDHB is one of four protein subunits that form succinate dehydrogenase, which catalyzes the oxidation of succinate [5455]. Two subunits of cytochrome c oxidase were also differentially expressed in oxidative phosphorylation. Cytochrome c oxidase is located at the end of cytochrome c system in cell respiration. This enzyme directly transfers the electron of respiratory substrate to molecular oxygen through cytochrome system [56-57].

It is widely acknowledged that steroid hormones play essential roles in sexual development. It is generally divided into five main classes, including glucocorticoids, mineralocorticoids, androgens, estrogens, and progestogens. Natural steroid hormones, which are lipids, are generally synthesized from cholesterol in the gonads and adrenal glands [58-59]. Hydroxysteroid dehydrogenase like 1 (HSDL1) was differentially expressed between CG vs SS and CG vs DS, indicating the expressions of HSDL1 were significantly regulated by the ablation of both single-side eyestalk and double-side eyestalk. HSDL1 was also reported to be involved in the mechanism of male sexual development in the previous study [38]. The shortchain dehydrogenase/reductases family (SDR) is a very large enzyme family, which can affect the mammalian reproduction, hypertension, neoplasia and digestion [60-61]. Hydroxysteroid dehydrogenase (HSD) is a subfamily of SDR, playing essential roles in sex-determination, the emergency and maintenance of the secondary sexual characters, and the regulation of endocrine 
through catalyzing the metabolism of steroid hormone. Hydroxysteroid dehydrogenase like 1 (HSDL1) was an important gene in the metabolic pathway of steroid hormone [62]. qPCR verification revealed that the expression pattern of important DEGs from these metabolic pathways were as the same as that of RNA-seq, indicating the accuracy of the transcriptome profiling analysis.

Both of this study and previous study predicted the potentially vital roles of HSDL1 in the mechanism of male sexual development in M. nipponense. Thus, the potential roles of HSDL1 in the male sexual development were also analyzed by using qPCR and RNAi, combined with the histological observations in this study. Previous studies revealed that HSDL1 was proven to be highly expressed in reproductive tissues (i.e., testis and ovary) in human, as revealed by northern blot analysis [62]. In situ hybridization indicated that the expression of HSDL1 is higher in the prostate cancer than that in the normal prostate. In addition, this gene is involved in the development of the sheep fetus in the late gestational stage [63]. The qPCR analysis in different mature tissues revealed that the highest expression level of Mn-HSDL1 was observed in hepatopancreas, followed by testis, while the Mn-HSDL1 RNA were rarely measured in other detected tissues [38]. Vitellogenin was reported to play essential roles in embryonic growth and gonadal development, which was only expressed in the female hepatopancreas, hemolymph, and ovary of M. nipponense [64]. The similar expression pattern of HSDL1 in the male prawns predicted that HSDL1 may play similar roles in the male sexual development of M. nipponense as that of vitellogenin in female sexual development. In the different developmental stages, the expressions in the larval developmental stages were generally higher than that of post-larval developmental stages, indicating HSDL1 was involved in the metamorphosis process of M. nipponense [65-66]. The Mn-HSDL1 mRNA expression was gradually increased from PL5 to PL25. The period from PL5 to PL25 was proven to be the sexdifferentiation sensitive period [5]. Thus, the increase from PL5 to PL25 indicated that HSDL1 
plays essential roles in gonad differentiation and development. In addition, the gender can be distinguished for the first time at PL25, and the expression in PL25 0 showed 2 times higher than that of PL25 9 , which also indicated that HSDL1 played more essential roles in male sexual development. The mRNA expressions of Mn-HSDL1 were significantly decreased at day 7 and day 14 after Mn-HSDL1 dsRNA injection, indicating the RNAi is efficient in this study. The mRNA expression of Mn-IAG was also measured in androgenic gland from the same prawn. The qPCR analysis revealed that the Mn-IAG expression was decreased with the decrease of Mn-HSDL1, indicating HSDL1 has positive regulatory effects on IAG in $M$. nipponense. IAG is a hormone, secreted by androgenic gland, promoting male sexual differentiation and development in many crustacean species [8-10]. Thus, HSDL1 was predicted to be involved in the male sexual development in $M$. nipponense. According to the histological observations, the number of sperms was decreased with the time of Mn-HSDL1 dsRNA injection. Compared with the control group, the sperms were rarely found at day 14 after Mn-HSDL1 dsRNA injection. This indicated that HSDL1 has positive regulatory effects on testis development in M. nipponense.

In conclusion, the potentially candidate genes involved in the male sexual development were selected through performing the long-reads and next generation transcriptome sequencing of androgenic gland after eyestalk ablation in M. nipponense. qPCR analysis revealed Mn-IAG was significantly increased after the ablation of both single-side and double-side eyestalk, indicating the ablation of eyestalk has dramatically regulatory roles on male sexual development in M. nipponense. The long-reads transcriptome generated 49,480 non-redundant transcripts. A total of 1,319, 2,092, 4,351 DEGs were identified between CG vs SS, SS vs DS, and CG vs DS, respectively, indicating the ablation of double-side eyestalk has more regulatory roles on male sexual development in M. nipponense. Cell cycle, Cellular senescence, Oxidative phosphorylation, Glycolysis/Gluconeogenesis and Steroid hormone biosynthesis were the 
main enriched metabolic pathways in all of these three comparisons, and the important DEGs from these metabolic pathways were identified. qPCR analysis and RNAi analysis of MnHSDL1 indicated that HSDL1 has positive regulatory effects on testis development. Overall, this study provided valuable resources for the researches of the mechanisms underlying male sexual development in M. nipponense and other crustacean species.

\section{Materials and Methods}

\section{Ethics statement}

The permission was obtained from the Tai Lake Fishery Management Council and the committee of Freshwater Fisheries Research Center during the experimental programs. MS222 anesthesia was used to sedate the prawns and shear the tissues.

\section{Sample collection}

A total of 600 healthy male prawns of $M$. nipponense were collected from a wild population in Tai Lake, Wuxi, China $\left(120^{\circ} 13^{\prime} 44^{\prime \prime} \mathrm{E}, 31^{\circ} 28^{\prime} 22^{\prime \prime} \mathrm{N}\right)$ with the body weights of 3.63-4.94 g. All the samples were randomly divided and transferred to three $500 \mathrm{~L}$ tanks and maintained in aerated freshwater for three days. The three groups were normal prawns (CG), single-side eyestalk ablation prawns (SS), double-side eyestalk ablation prawns (DS). The androgenic glands were respectively collected from these three groups after 7 days of eyestalk ablation, and immediately preserved in liquid nitrogen until used for long-reads and next-generation transcriptomic analysis. Different mature tissues included testis, ovary, hepatopancreas, muscle, eyestalk, gill, heart and brain. Specimens for the different stages of larval and post-larval developmental stages were from the full-sibs population, collected with their maturation process.

\section{Long-reads transcriptome analysis}

In order to provide sufficient RNA with an aim to establish a reference transcriptome for further analysis, equal amount of androgenic gland of CG, SS and DS (N $\geq 60)$ were pooled together 
to perform the long-reads sequencing. According to the manufacturer's instructions, UN1Q-10 Column Trizol Total RNA Isolation Kit (Sangon, Shanghai, China) was used to extract total RNA, and an Agilent RNA 6000 Nano kit and chips on a Bioanalyzer 2100 (Agilent Technologies, Santa Clara, CA, USA) were used to measure the RNA integrity. A PacBio RSII platform (Pacific Bioscience Inc., Menlo Park, CA, USA) was employed to construct the longreads transcriptome. The detailed procedures for the construction of long-reads transcriptome and the analysis of raw sequence data have been well described in our previous study [67].

In the subsequent step, the contaminant sequences were removed by stepwise CLC [68], and the LRS isoforms were annonated [69]. Using Blastp, the transcriptome factors were aligned to the PlnTFDB database (http://plntfdb.bio.uni-potsdam.de/v3.0/), the AnimalTFDB database (http://bioinfo.life.hust.edu.cn/AnimalTFDB/), and the CARD database (https://card.mcmaster.ca/) for selection of genes, involved in the mechanism of male sexual development in M. nipponense, using the threshold of E-value $\geq 1 \mathrm{e}^{-10}$. Finally, all Blastp results were processed with BLAST2GO [70] for functional annotation.

\section{Transcriptomic profiling analysis}

The comparative transcriptome analysis of androgenic gland between the CG, SS, and DS were performed. In order to ensure the sufficient amount of RNA samples, androgenic gland from at least 30 prawns were pooled to form one biological replicate, and three biological replicates were sequenced for all of these three groups. The previously published studies have been well described the experimental process $[12,36]$.

Clean reads were assembled into non-redundant transcripts by using Trinity program (version: trinityrnaseq_r20131110) [71]. The NR protein, the Gene Ontology (GO), the Cluster of Orthologous Groups (COG), and the Kyoto Encyclopedia of Genes and Genomes (KEGG) database were then used to perform the gene annotation, using an E-value cut-off of $10^{-5}$ [12]. Blast2go software was used for functional annotation by GO terms [70]. Blast software was 
employed to perform the functional annotation against the COG [72] and KEGG [73] database. EB-seq algorithm was used to filter the differentially expressed genes, under the criteria of FDR (False discovery rate) $<0.05[74]$.

\section{qPCR analysis}

qPCR was used to measure the relative mRNA expressions of Mn-HSDL1 in different developmental stages, and qPCR verification of important DEGs. The Bio-Rad iCycler iQ5 Real-Time PCR System (Bio-Rad) was used to carry out the SYBR Green RT-qPCR assay. The procedure has been well described in details in previous studies [17-18]. The primers used for qPCR analysis were listed in Table 1 . The primers used for qPCR verification of important DEGs were listed in Table 2. EIF was used as reference gene in this study [75].

\section{RNA interference (RNAi) analysis}

RNAi was performed to analysis the potentially regulatory roles on Mn- HSDL1 in male sexual development in M. nipponense. Snap Dragon tools was used to design the specific RNAi primer with $\mathrm{T} 7$ promoter site (http://www.flyrnai.org/cgibin/RNAifind_primers.pl), and shown in Table 1. The Transcript Aid ${ }^{\mathrm{TM}} \mathrm{T} 7 \mathrm{High}$ Yield Transcription kit (Fermentas, Inc, USA) was used to synthesize the Mn-HSDL1 dsRNA, followed by the procedures of the manufacturer. A total of 300 health mature male M. nipponense were collected with body weight of $3.21-4.78 \mathrm{~g}$, and divided into two groups. As described in previous study [76-77], the prawns from experimental group were injected with $4 \mu \mathrm{g} / \mathrm{g}$ Mn- HSDL1 dsRNA, while the prawns from control group were injected with equal volume of GFP. The HSDL1 Mrna expression were investigated in the androgenic gland by qPCR after the injection of 1, 7 and 14 days, in order to detect the interference efficiency ( $\mathrm{N} \geq 5$ ). The Mrna expressions of Mn-IAG were also measured in the same Cdna templates, in order to analysis the regulatory relationship between Mn-HSDL1 and Mn-IAG.

\section{Histological observation}


The morphological changes of the testis between different days after RNAi treatment was observed by Hematoxylin and eosin (HE) staining. Five testicular samples were respectively collected after 1, 7, and 14 days of RNAi treatment for HE staining. The procedures have been well described in previous studies [78-79]. Olympus SZX16 microscope was used to observe the slides (Olympus Corporation, Tokyo, Japan). The various cell types were labelled based on morphological analysis [5].

\section{Statistical Analysis}

Quantitative data were expressed as mean \pm SD. Statistical differences were estimated by oneway ANOVA followed by LSD and Duncan's multiple range test. All statistics were measured using SPSS Statistics 23.0. A probability level of 0.05 was used to indicate significance $(p<$ $0.05)$.

\section{Additional files}

Table S1: Summary of BLASTx results for unigenes of androgenic gland long-reads $M$. nipponense transcriptome.

Acknowledgements: This research was supported by grants from the National Key R\&D Program of China (2018YFD0900201); Central Public-interest Scientific Institution Basal Research Fund CAFS (2019JBFM02, 2019JBFM04); the National Key R\&D Program of China (2018YFD0901303); Jiangsu Agricultural Industry Technology System (JFRS-02); the National Natural Science Foundation of China (31572617); the China Agriculture Research System-48 (CARS-48); the New cultivar breeding Major Project of Jiangsu province (PZCZ201745).

\section{Available data and materials}

The reads of $M$. nipponense transcriptome were submitted to NCBI with the accession number of PRJNA533885.

\section{Reference}


1. Cai, Y., \& Shokita, S. Report on a collection of freshwater shrimps (Crustacea: Decapoda: Caridea) from the Philippines, with descriptions of four new species. Raffles B. Zool. 54, $245-270$ (2006).

2. Ma, K.Y., Feng, J.B., Lin, J.Y., \& Li, J.L. The complete mitochondrial genome of Macrobrachium nipponense. Gene 487, 160-165 (2011).

3. Salman, S.D., Page, T.J., Naser, M.D., \& Yasser, A.G. The invasion of Macrobrachium nipponense (De Haan, 1849) (Caridea: Palaemonidae) into the southern Iraqi marshes. Aquat. Invasions 1, 109-115 (2006).

4. Wang, Y.B. et al. Identification and Characterization of the DMRT11E Gene in the Oriental River Prawn Macrobrachium nipponense. Int. J. Mol. Sci. 20, 1734 (2019).

5. Jin, S.B. et al. Histological observation of gonadal development during post-larva in oriental river prawn, Macrobrachium nipponense, Chin. J. Fish. 29, 11-16 (2016).

6. Sagi, A., Cohen, D., \& Wax, Y. Production of Macrobrachium rosenbetgii in momosex population: yield characteristes under intensive monoculture conditions in cages. Aquaculture 51, 265-275 (1986).

7. Sagi, A., Cohen, D., \& Milner, Y. Effect of androgenic gland ablation on morphotypic differentiation and sexual characteristics of male freshwater prawns, Macrobrachium rosenbergii. Gen. Comp. Endocr. 77, 15-22 (1990).

8. Rosen, O. et al. A sexual shift induced by silencing of a single insulin-like gene in crayfish: ovarian upregulation and testicular degeneration. PLoS One 5, e15281 (2010).

9. Ventura, T. et al. Temporal silencing of an androgenic gland-specific insulin-like gene affecting phenotypical gender differences and spermatogenesis. Endocrinology 150, 12781286 (2009). 
10. Ventura, T. et al. Expression of an androgenic gland-specific insulin-like peptide during the course of prawn sexual and morphotypic differentiation. ISRN Endocrinol. 2011, $476283(2011)$.

11. Ventura, T. et al. Timing sexual differentiation: full functional sex reversal achieved through silencing of a single insulin-like gene in the prawn, Macrobrachium rosenbergii. Biol. Reprod. 86, 90 (2012).

12. Jin, S.B. et al. Transcriptome analysis of androgenic gland for discovery of novel genes from the oriental river prawn, Macrobrachium nipponense, using Illumina Hiseq 2000. PLOS ONE 8, e76840 (2013).

13. Jin, S.B. et al. Identification of androgenic gland microRNAs and their targeted genes for discovery of sex-related microRNAs in oriental river prawn, Macrobrachium nipponense. Genet. Mol. Res. 14, 18396-18406 (2015).

14. Jin, S.B. et al. iTRAQ-based quantitative proteomic analysis of the androgenic glands of the oriental river p.rawn, Macrobrachium nipponense, during nonreproductive and reproductive seasons. Comp. Biochem. Phys. D 26, 50-57 (2018).

15. Jin, S.B. et al. Potential functions of Gem-associated protein 2-like isoform X1 in the oriental river prawn Macrobrachium nipponense: Cloning, qPCR, in situ hybridization, and RNAi analysis. Int. J. Mol. Sci. 20, 3995 (2019).

16. Jin, S.B. et al. Identification of potentially novel functions of DNA polymerase zeta catalytic subunit in oriental river prawn, Macrobrachium nipoponense: Cloning, qPCR, in situ hybridization and RNAi analysis. 3 Biotech 9, 330 (2019).

17. Jin, S.B. et al. Molecular Cloning, Expression, and In situ Hybridization Analysis of Forkhead box protein L2 during Development in Macrobrachium nipponense. J. World Aquacult. Soc. 49, 429-440 (2018).

18. Jin, S.B. et al. Molecular cloning of two tropomyosin family genes and expression analysis 
during development in oriental river prawn, Macrobrachium nipponense. Gene 546, 390397 (2014).

19. Hopkins, P.M. The eyes have it: a brief history of crustacean neuroendocrinology. Gen. Comp. Endocrinol. 175, 357-366 (2012).

20. Treerattrakool, S., Panyim, S., \& Udomkit, A. Induction of ovarian maturation and spawning in Penaeus monodon broodstock by double-stranded RNA. Mar. Biotechnol. 13, 163-169 (2011).

21. Treerattrakool, S., Chartthai, C., Phromma-in, N., Panyim, S., \& Udomkit, A. Silencing of gonad-inhibiting hormone gene expression in Penaeus monodon by feeding with GIH dsRNA-enriched Artemia. Aquaculture 404, 116-121 (2013).

22. Revathi, P. et al. Impact of eyestalk ablation on the androgenic gland activity in the freshwater prawn Macrobrachium rosenbergii (De Man). World 5, 373-381 (2013).

23. Pamuru, R.R. et al. Stimulation of molt by RNA interference of the molt inhibiting hormone in the crayfish Cherax quadricarinatus. Gen. Comp. Endocrinol. 178, 227-236 (2012).

24. Salma, U. et al. Five hepatopancreatic and one epidermal chitinases froma pandalid shrimp (Pandalopsis japonica): cloning and effects of eyestalk ablation on gene expression. Comp. Biochem. Phys. B 161, 197-207 (2012).

25. Shen, H., Zhou, X., Bai, A., Ren, X., \& Zhang, Y. Ecdysone receptor gene from the freshwater prawn Macrobrachium nipponense: identification of different splice variants and sexually dimorphic expression, fluctuation of expression in the molt cycle and effect of eyestalk ablation. Gen. Comp. Endocrinol. 193, 86-94 (2013).

26. Santos, E.A., Eduardo, L., Nery, M., Goncalves, A.A., \& Keller, R. Evidence for the involvement of the crustacean hyperglycemic hormone in the regulation of lipid metabolism. Physiol. Biochem. Zool. 70, 415-420 (1997). 
27. Almeida, E.A., Petersen, R.L., Andreatta, E.R., \& Bainy, A.C. Effects of captivity and eyestalk ablation on antioxidant status of shrimps (Farfantepenaeus paulensis), Aquaculture 238, 523-528 (2004).

28. Tiu, S.H.K., \& Chan, S.M. The use of recombinant protein and RNA interference approaches to study the reproductive functions of a gonad-stimulating hormone from the shrimp Metapenaeus ensis. FEBS J. 274, 4385-4395 (2007).

29. Sainz-Hernández, J.C., Racotta, I.S., Dumas, S., \& Hernández-López, J. Effect of unilateral and bilateral eyestalk ablation in Litopenaeus vannamei male and female on several metabolic and immunologic variables. Aquaculture 283, 188-193 (2008).

30. Diarte-Plata, G. et al. Eyestalk ablation procedures tominimize pain in the freshwater prawn Macrobrachium Americanum. Appl. Anim. Behav. Sci. 140, 172-178 (2012).

31. Qiao, H. et al. Characterization, expression, and function analysis of gonad-inhibiting hormone in Oriental River prawn, Macrobrachium nipponense and its induced expression by temperature. Comp. Biochem. Phys. A. 185, 1-8 (2015).

32. Qiao, H. et al. Characterization, expression patterns of molt-inhibiting hormone gene of Macrobrachium nipponense and its roles in molting and growth. PloS one 13, e0198861 (2018).

33. Jin, S.B. et al. Molecular cloning and expression of a full-length cDNA encoding crustacean hyperglycemic hormone $(\mathrm{CHH})$ in oriental river pawn (Macrobrachium nipponense). J. Fish. China. 20, 82-92 (2013).

34. Chung, J.S., Manor, R., \& Sagi, A. Cloning of an insulin-like androgenic gland factor (IAG) from the blue crab, Callinectes sapidus: implications for eyestalk regulation of IAG expression. Gen. Comp. Endocrinol. 173, 4-10 (2011). 
35. Sroyraya, M. et al. Bilateral eyestalk ablation of the blue swimmer crab, Portunus pelagicus, produces hypertrophy of the androgenic gland and an increase of cells producing insulinlike androgenic gland hormone. Tissue Cell 42, 293-300 (2010).

36. Guo, Q. Li, S. \& Lv, X. Sex-Biased CHHs and Their Putative Receptor Regulate the Expression of IAG Gene in the Shrimp Litopenaeus vannamei. Front. Physiol. 10, 1525 (2019).

37. Jin, S.B., et al. Integrated analysis of microRNA and mRNA expression profiles at sexdifferentiation sensitive period in oriental river prawn, Macrobrachium nipponense. Sci. Rep. 7, 12011 (2017).

38. Jin, S. et al. Analysis of testis metabolome and transcriptome from the oriental river prawn (Macrobrachium nipponense) in response to different temperatures and illumination times. Comp. Biochem. Phys. D 34, 100662 (2020).

39. Spellman, P.T. et al. Comprehensive identification of cell cycle-regulated genes of the yeast Saccharomyces cerevisiae by microarray hybridization. Mol. Biol. Cell. 9, 3273-97 (1998).

40. Kamb, A. A cell cycle regulator potentially involved in genesis of many tumour types. Trend Genet. 10, 228 (1994).

41. Barrett, J.C. et al. Cellular senescence and cancer. Cold Spring Harb Symp Quant Biol. 59, 411-8 (1994).

42. Chen, Z.B. et al. Crucial role of p53-dependent cellular senescence in suppression of Ptendeficient tumorigenesis. Nature 43, 6725-30 (2005).

43. Russo, A.A., Jeffrey, P.D., Patten, A.K., Massagué, J., \& Pavletich, N.P. Crystal structure of the p27Kip1 cyclin-dependent-kinase inhibitor bound to the cyclin A-Cdk2 complex. Nature 382, 325-31 (1996).

44. Girard, F., Strausfeld, U., Fernandez, A., \& Lamb, N.J. Cyclin A is required for the onset 
of DNA replication in mammalian fibroblasts. Cell 67, 1169-79 (1991).

45. Lees, E., Faha, B., Dulic, V., Reed, S.I., \& Harlow, E. Cyclin E/cdk2 and cyclin A/cdk2 kinases associate with p107 and E2F in a temporally distinct manner. Genes Dev. 6, $1874-85$ (1992).

46. King, R.W. et al. A 20S complex containing CDC27 and CDC16 catalyzes the mitosisspecific conjugation of ubiquitin to cyclin B. Cell 81, 279-88 (1995).

47. Kadyrova, L.Y., Habara, Y., Lee, T.H., \& Wharton, R.P. Translational control of maternal Cyclin B mRNA by Nanos in the Drosophila germline. Development 134, 1519-27 (2007).

48. Huang, J., \& Raff, J.W. The disappearance of cyclin B at the end of mitosis is regulated spatially in Drosophila cells. MBO J. 18, 2184-95 (1999).

49. Gu, Y., Turck, C.W., Morgan, D.O. Inhibition of CDK2 activity in vivo by an associated 20K regulatory subunit. Nature 366, 707-10 (1993).

50. Koff, A. et al. Formation and activation of a cyclin E-cdk2 complex during the G1 phase of the human cell cycle. Science 257, 1689-94 (1992).

51. Clurman, B.E., Sheaff, R.J., Thress, K., Groudine, M., \& Roberts, J.M. Turnover of cyclin E by the ubiquitin-proteasome pathway is regulated by cdk2 binding and cyclin phosphorylation. Genes Dev. 10, 1979-90 (1996).

52. Dimroth, P., Kaim, G., \& Matthey, U. Crucial role of the membrane potential for ATP synthesis by F(1)F(o) ATP synthases. J. Exp. Biol. 203, 51-59 (2000).

53. Lubert, S. "Glycolysis," in Biochemistry, 4th Edn. (New York, NY: W.H. Freeman and Company), 483-508 (1995).

54. Au, H.C. et al. Structural organization of the gene encoding the human iron-sulfur subunit of succinate dehydrogenase. Gene 159, 249-53 (1995).

55. Kita, K., Oya, H., Gennis, R.B., Ackrell, B.A., \& Kasahara, M. Human complex II 
(succinate-ubiquinone oxidoreductase): cDNA cloning of iron sulfur (Ip) subunit of liver mitochondria. Biochem. Biophys. Res. Commun. 166, 101-8 (1990).

56. Gurumoorthy, P., \& Ludwig, B. Deciphering protein-protein interactions during the biogenesis of cytochrome c oxidase from Paracoccus denitrificans. FEBS J. 282, 53749 (2015).

57. Cleeter, M.W. Cooper, J.M., Darley-Usmar, V.M., Moncada, S., \& Schapira, A.H. Reversible inhibition of cytochrome c oxidase, the terminal enzyme of the mitochondrial respiratory chain, by nitric oxide. Implications for neurodegenerative diseases. FEBS Lett. 345, 50-4 (1994).

58. Mayes, J.S., \& Watson, G.H. Direct effects of sex steroid hormones on adipose tissues and obesity. Obes. Rev. 5, 197-216 (2010).

59. Payne, A.H., \& Hales, D.B. Overview of steroidogenic enzymes in the pathway from cholesterol to active steroid hormones. Endocr. Rev. 25, 947-70 (2004).

60. Kallberg, Y., Oppermann, U., Jörnvall, H., \& Persson, B. Short-chain dehydrogenases/reductases (SDRs), Eur. J. Biochem. 268 (2002) 4409-4417.

61. Jörnvall, H. et al. Short-chain dehydrogenases/reductases (SDR). Biochemistry 34, 6003$13(1995)$.

62. Huang, Y. et al. A novel human hydroxysteroid dehydrogenase like 1 gene (HSDL1) is highly expressed in reproductive tissues. Mol. Biol. Rep. 28, 185-191 (2002).

63. Gentili, S., Morrison, J.L., \& McMillen, I.C. Intrauterine growth restriction and differential patterns of hepatic growth and expression of IGF1, PCK2, and HSDL mRNA in the sheep fetus in late gestation. Biol. Reprod. 80, 1121-7 (2009).

64. Bai, H.K. et al. Molecular characterization and developmental expression of vitellogenin in the oriental river prawn Macrobrachium nipponense and the effects of RNA 
interference and eyestalk ablation on ovarian maturation. Gene 562, 22-31 (2015).

65. Zhang, Y.P. et al. Molecular cloning and expression analysis of transformer-2 gene during development in Macrobrachium nipponense (de Haan 1849). J. World Aquacult. Soc. 44, 338-349 (2013).

66. Zhang, Y.P. et al. Molecular cloning and expression analysis of extra sex combs gene during development in Macrobrachium nipponense. Turk. J. Fish. Aquat. Sc. 13, 331-340 (2013).

67. Jin, S. et al. Identification of candidate genes for the plateau adaptation of a Tibetan amphipod, Gammarus lacustris, through integration of genome and transcriptome sequencing. Front. Genet. 10 (2019).

68. Cheng, B., Furtado, A., Henry, R. Processing of Pacbio Isoseq sequences. protocols.io $2017 \mathrm{a}$.

69. Cheng, B., Furtado, A., Henry, R. Transcriptome annotation. protocols.io $2017 \mathrm{~b}$.

70. Ashburner, M. et al. Gene ontology: tool for the unification of biology. Nat. Genet. 25, 259 (2000).

71. Grabherr, M.G. et al. Trinity: reconstructing a full-length transcriptome without a genome from RNA-Seq data. Nat. Biotechnol. 29, 644-652 (2011).

72. Tatusov, R.L. et al. The COG database: an updated version includes eukaryotes. $B M C$ Bioinformatics. 4, 41 (2003).

73. Minoru, K.A. et al. KEGG for linking genomes to life and the environment. Nucleic. Acids. Res. 36, D480-D484 (2008).

74. Benjamini, Y., Drai, D., Elmer, G., Kafkafi, N., \& Golani, L. Controlling the false discovery rate in behavior genetics research. Behav. Brain. Res. 125, 279-284 (2001).

75. Hu, Y.N. et al. Validation and evaluation of reference genes for Quantitative real-time PCR in Macrobrachium nipponense. Int. J. Mol. Sci. 19, 2258 (2018). 
627

628

629

630

631

632

633

634

635

636

637

638

639

\section{7}

76. Li, F. et al. Identification and characterization of opsin gene and its role in ovarian maturation in the oriental river prawn Macrobrachium nipponense. Comp. Biochem. Physiol. B. 218, 1-12 (2018).

77. Jiang, F.W. et al. The RNA Interference Regularity of Transformer-2 Gene of Oriental River Prawn Macrobrachium nipponense. Chin. Agricult. Sci. Bul. 30, 32-37 (2014).

78. Ma, X.K., Liu, X.Z., Wen, H.S., Xu, Y.J., \& Zhang, L.J. Histological observation on gonadal sex differentiation in Cynoglossus semilaevis Günther. Mar. Fish. Res. 27, 55-61 (2006).

79. ShangGuan, B.M. Liu, Z.Z., \& Li, S.Q. Histological Studies on Ovarian Development in Scylla serrata. J. Fish. China. 15, 96-103 (1991).

6

7

8

9

0

1

2

3

34

35

36

9 
Figure 1: Measurement of the expression of Mn-IAG after the ablation of eyestalk. The amount of Mn-IAG mRNA was normalized to the EIF transcript level. Data are shown as mean +SD (standard deviation) of tissues from three separate individuals. Capital letters indicated expression difference between different days in the same group. $*(p<0.05)$ and $* *(p<0.01)$ indicates significant expression difference between different groups at the sample day.

Figure 2: Gene ontology classification of non-redundant transcripts. By alignment to GO terms, 19,673 unigenes were mainly divided into three categories with 52 functional groups: biological process (19 functional groups), cellular component (16 functional groups), and molecular function (17 functional groups). The left y-axis indicates the percentage of a specific category of genes existed in the main category, whereas the right y-axis indicates the number of a specific category of genes existed in main category.

Figure 3: Cluster of orthologous groups (COG) classification of putative proteins.

Figure 4: Verification of the expressions of 10 differentially expressed genes (DEGs) between the androgenic gland of normal prawns, prawns of single-side ablation and prawns of doubleside ablation by qPCR. The amounts of DEGs expression were normalized to the EIF transcript level. Data are shown as mean $\pm \mathrm{SD}$ (standard deviation) of tissues in three separate individuals. Capital letter indicates expression.

Figure 5: Expression characterization of Mn-HSDL1 in different developmental stages. The amount of Mn-HSDL1 mRNA was normalized to the EIF transcript level. Data are shown as mean + SD (standard deviation) of tissues from three separate individuals. Capital letters indicate expression difference between different samples.

Figure 6: Expression characterization of Mn-HSDL1 and Mn-IAG at different days after MnHSDL1 dsRNA injection. The amount of Mn-HSDL1 and Mn-IAG mRNA was normalized to the EIF transcript level. Data are shown as mean +SD (standard deviation) of tissues from three separate individuals. Capital letters indicated expression difference between different days after 
666 GFP injection in control group. Lowercase indicated expression difference between different 667 days after Mn-HSDL1 dsRNA injection in RNAi group. $*(p<0.05)$ and $* *(p<0.01)$ indicates 668 significant expression difference between the RNAi group and control group at the sample day. 669 A: Expression characterization of Mn-HSDL1 at different days after Mn-HSDL1 dsRNA 670 injection. B: Expression characterization of Mn-IAG at different days after Mn-HSDL1 dsRNA 671 injection.

672 Figure 7: The histological observations of testis between RNAi and control group. SG: Spermatogonia; SC: spermatocyte; S: sperm; CT: collecting tissue. Scale bars $=20 \mu \mathrm{m}$. 
Table 1. Primers used for $\mathrm{qPCR}$ verification

\begin{tabular}{ll}
\hline Primer & Sequence \\
\hline Cyclin B3-F & TGATGAAAGAACTCCGCCGT \\
Cyclin B3-R & AGCGCACCTGGCATATCTTC \\
MAD2A-F & ACCCTCCTGAGTCCTTCACTT \\
MAD2A-R & TGCACATGTCCTGCCTCAAG \\
Polo-F & CGAACTACATCGCCCCAGAA \\
Polo-R & AGCGGTCCAATTCTCGAAGG \\
Cyclin A-F & CTGCCTCATCAGTTGCGTTG \\
Cyclin A-R & AGCTGTGATACCGAATGCCA \\
Cdc2-F & ATCAGCGCAGAGTTCTTCACA \\
Cdc2-R & GAAGAACTTCAGGTGCACGG \\
Pyclin B-F & TGGGAGATGTGGGAAATCGG \\
Cyclin B-R & CCTCAACCTTCGCTTCTTGC \\
Estrogen-F & CTGCAAAACTGGCGGTCAAA \\
Estrogen-R & CGAGACCTGGGACGTCATTC \\
Alcohol-F & CCTTCCTCCAGGGACTCGTA \\
\hline
\end{tabular}


Table 2. Primers used for HSDL1 analysis

\begin{tabular}{lll}
\hline Primer name & Nucleotide Sequence $\left(5^{\prime} \rightarrow 3^{\prime}\right)$ & Purpose \\
\hline HSDL1-RTF & AGCCTAAGCGTTCCAACTCC & FWD primer for GEM expression \\
HSDL1-RTR & TATTCAGCAGACCTCGTGGC & RVS primer for $G E M$ expression \\
EIF-F & CATGGATGTACCTGTGGTGAAAC & FWD primer for $\beta$-actin expression \\
EIF-R & CTGTCAGCAGAAGGTCCTCATTA & RVS primer for $\beta$-actin expression \\
HSDL1 RNAi-F & TAATACGACTCACTATAGGGGCAGACTTCTCCAACGGAAG & FWD primer for RNAi analysis \\
HSDL1 RNAi-R & TAATACGACTCACTATAGGGGCAGAGCTTAACGGATGAGG & RVS primer for RNAi analysis \\
\hline
\end{tabular}


Table 3. Identification of important DEGs from transcriptome profiling analysis

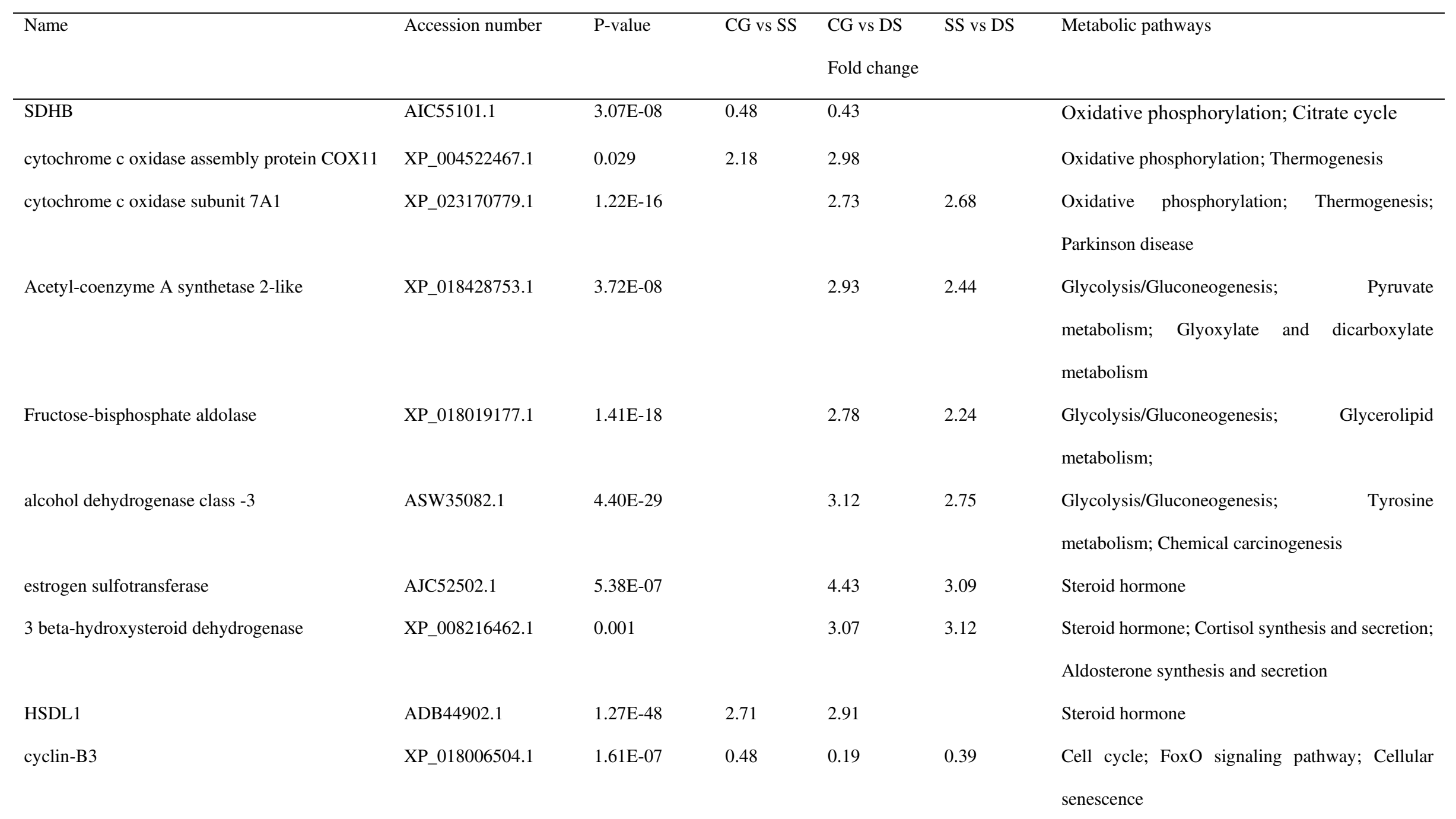




\begin{tabular}{|c|c|c|c|c|c|c|}
\hline MAD2A-like & XP_023320668.1 & $1.09 \mathrm{E}-13$ & 0.45 & 0.17 & 0.37 & $\begin{array}{l}\text { Cell cycle; Progesterone-mediated oocyte } \\
\text { maturation; Oocyte meiosis }\end{array}$ \\
\hline polo-like kinase 1 & AMO03195.1 & $5.47 \mathrm{E}-18$ & 0.33 & 0.08 & 0.24 & $\begin{array}{l}\text { Cell cycle; FoxO signaling pathway; } \\
\text { Progesterone-mediated oocyte maturation; Oocyte } \\
\text { meiosis }\end{array}$ \\
\hline cyclin A & AGG40744.1 & $1.21 \mathrm{E}-15$ & 0.49 & 0.15 & 0.31 & $\begin{array}{l}\text { Cell cycle; Human papillomavirus infection; } \\
\text { Epstein-Barr virus infection; Progesterone- } \\
\text { mediated oocyte maturation; Cellular senescence }\end{array}$ \\
\hline Cdc2 kinase & ADB44904.1 & $1.87 \mathrm{E}-27$ & 0.45 & 0.13 & 0.29 & $\begin{array}{l}\text { Cell cycle; Gap junction; Oocyte meiosis; p53 } \\
\text { signaling pathway; Cellular senescence }\end{array}$ \\
\hline cyclin B & ADB44902.1 & $8.92 \mathrm{E}-32$ & 0.37 & 0.10 & 0.26 & $\begin{array}{l}\text { Cell cycle; Progesterone-mediated oocyte } \\
\text { maturation; Oocyte meiosis; FoxO signaling } \\
\text { pathway; Cellular senescence; p53 signaling } \\
\text { pathway }\end{array}$ \\
\hline
\end{tabular}




\section{Figures}

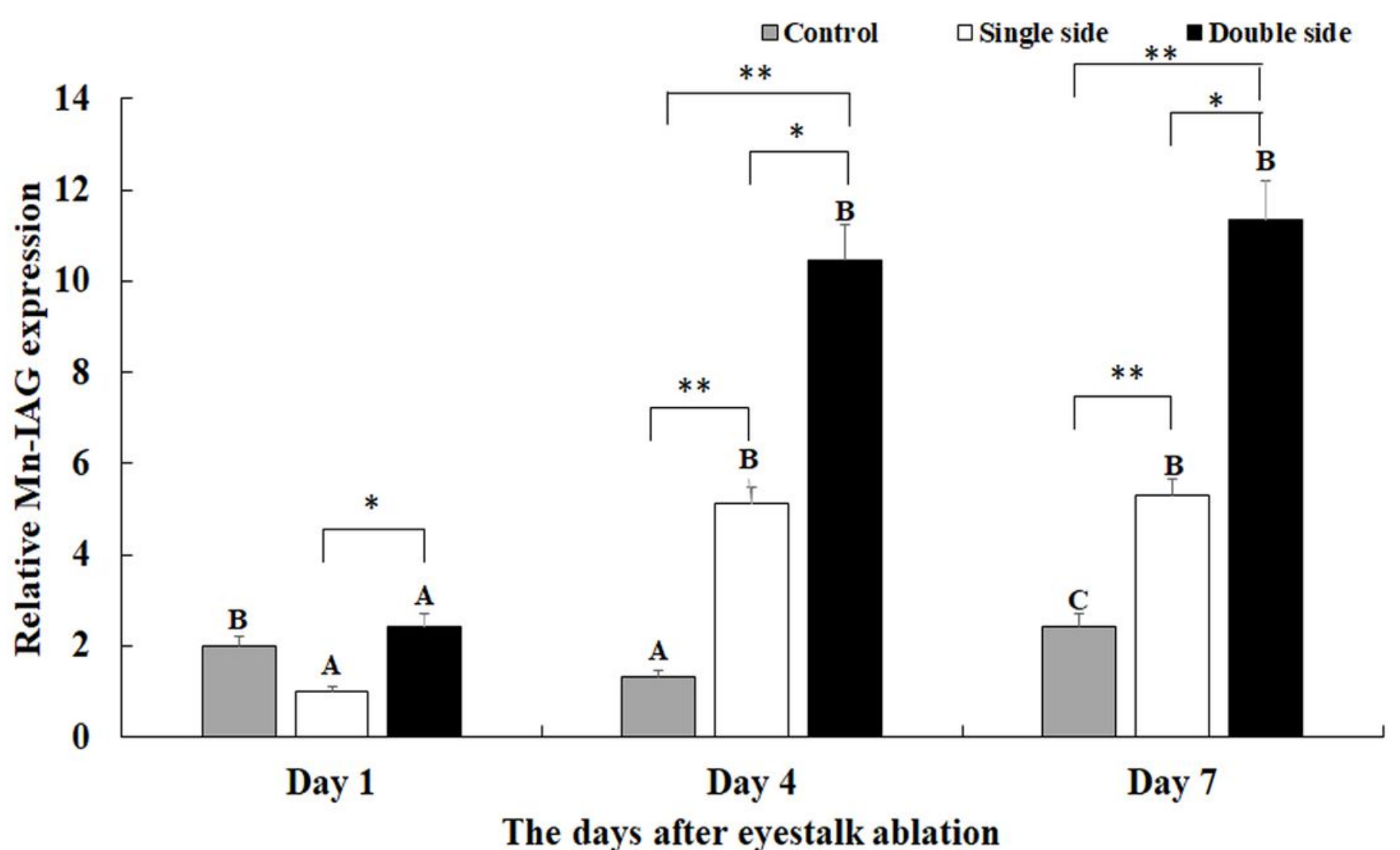

Figure 1

Measurement of the expression of Mn-IAG after the ablation of eyestalk. The amount of Mn-IAG mRNA was normalized to the EIF transcript level. Data are shown as mean +SD (standard deviation) of tissues from three separate individuals. Capital letters indicated expression difference between different days in the same group. ${ }^{*}(p<0.05)$ and $* *(p<0.01)$ indicates significant expression difference between different groups at the sample day. 


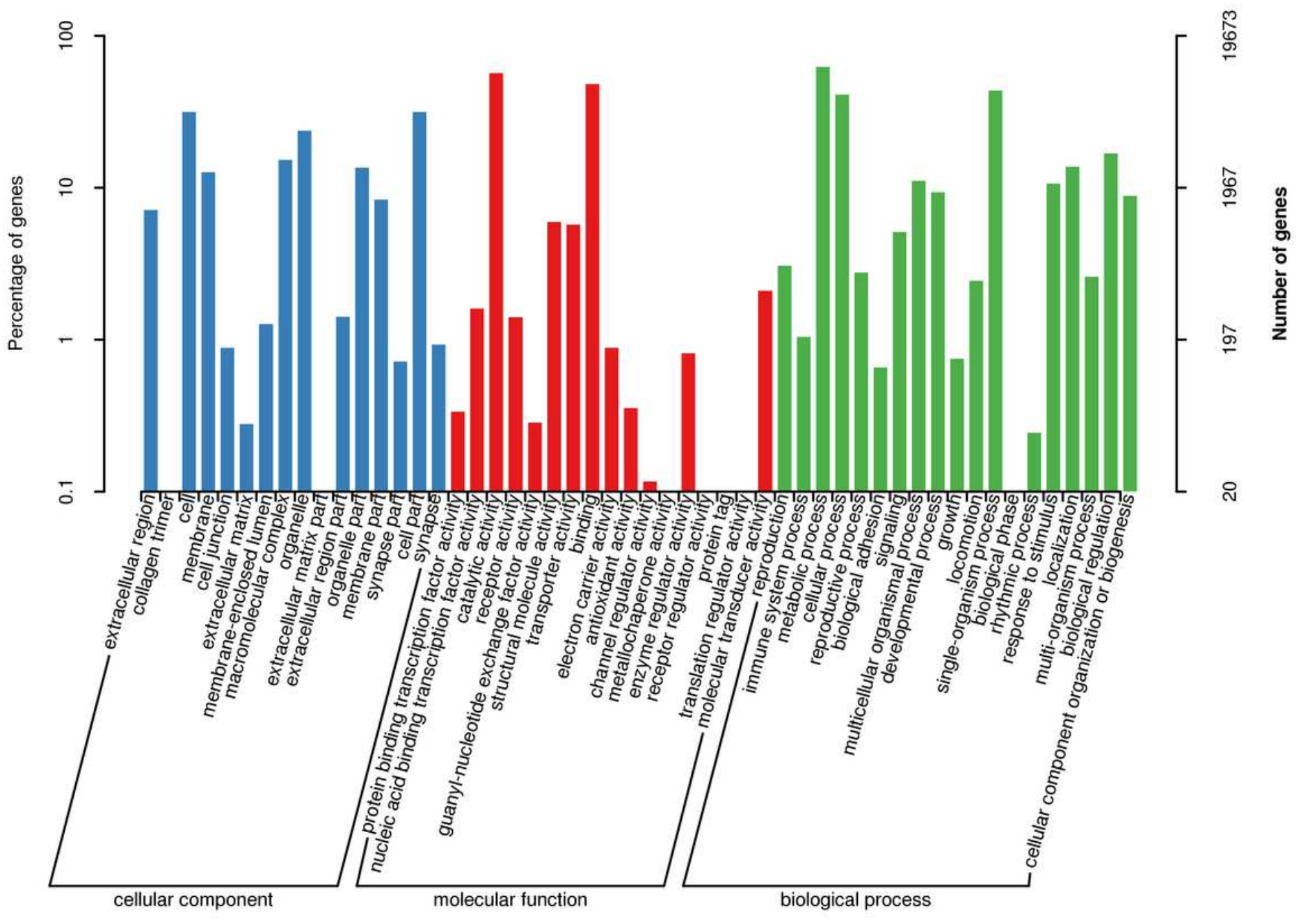

Figure 2

Gene ontology classification of non-redundant transcripts. By alignment to GO terms, 19,673 unigenes were mainly divided into three categories with 52 functional groups: biological process ( 19 functional groups), cellular component (16 functional groups), and molecular function (17 functional groups). The left $y$-axis indicates the percentage of a specific category of genes existed in the main category, whereas the right $y$-axis indicates the number of a specific category of genes existed in main category. 


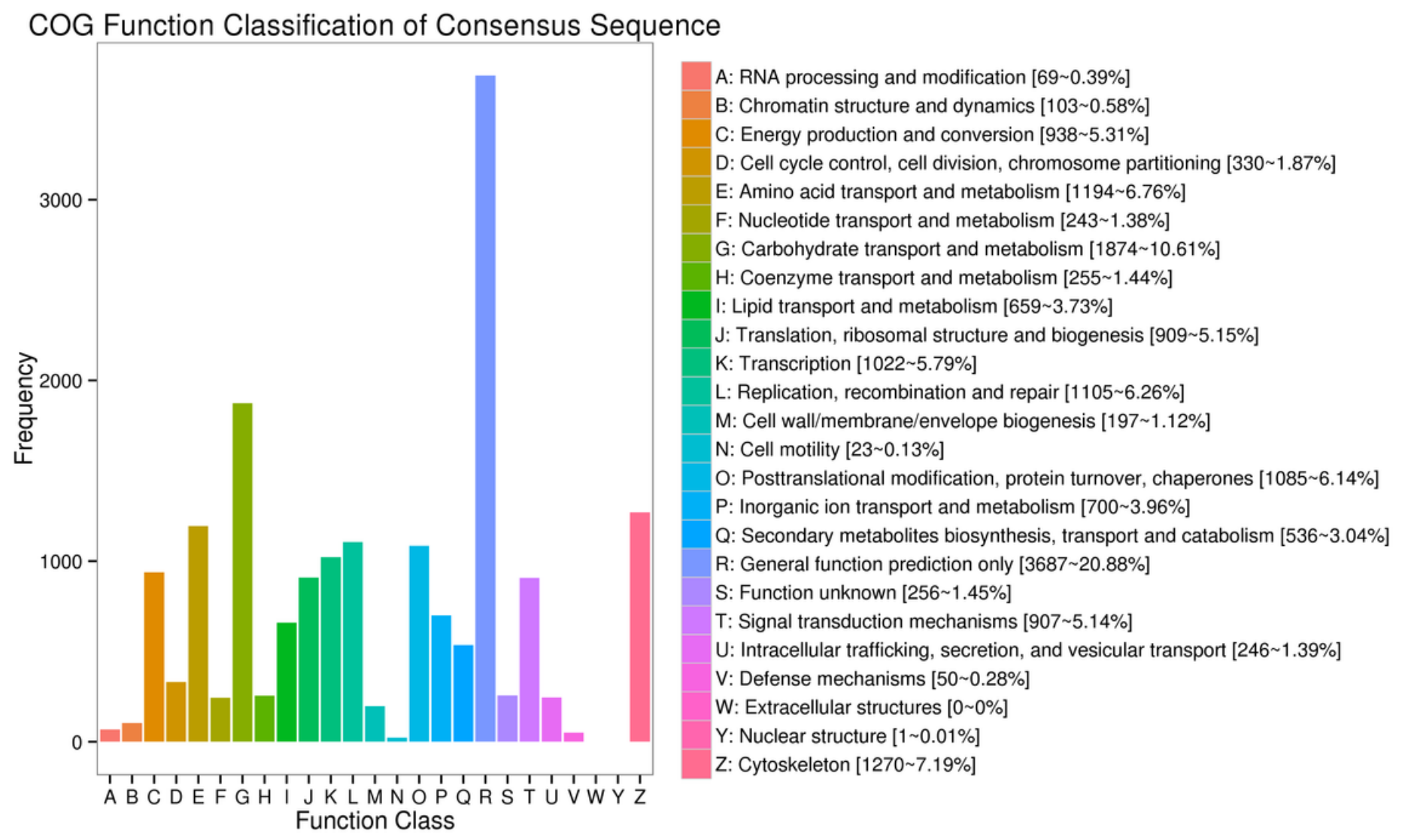

Figure 3

Cluster of orthologous groups (COG) classification of putative proteins. 

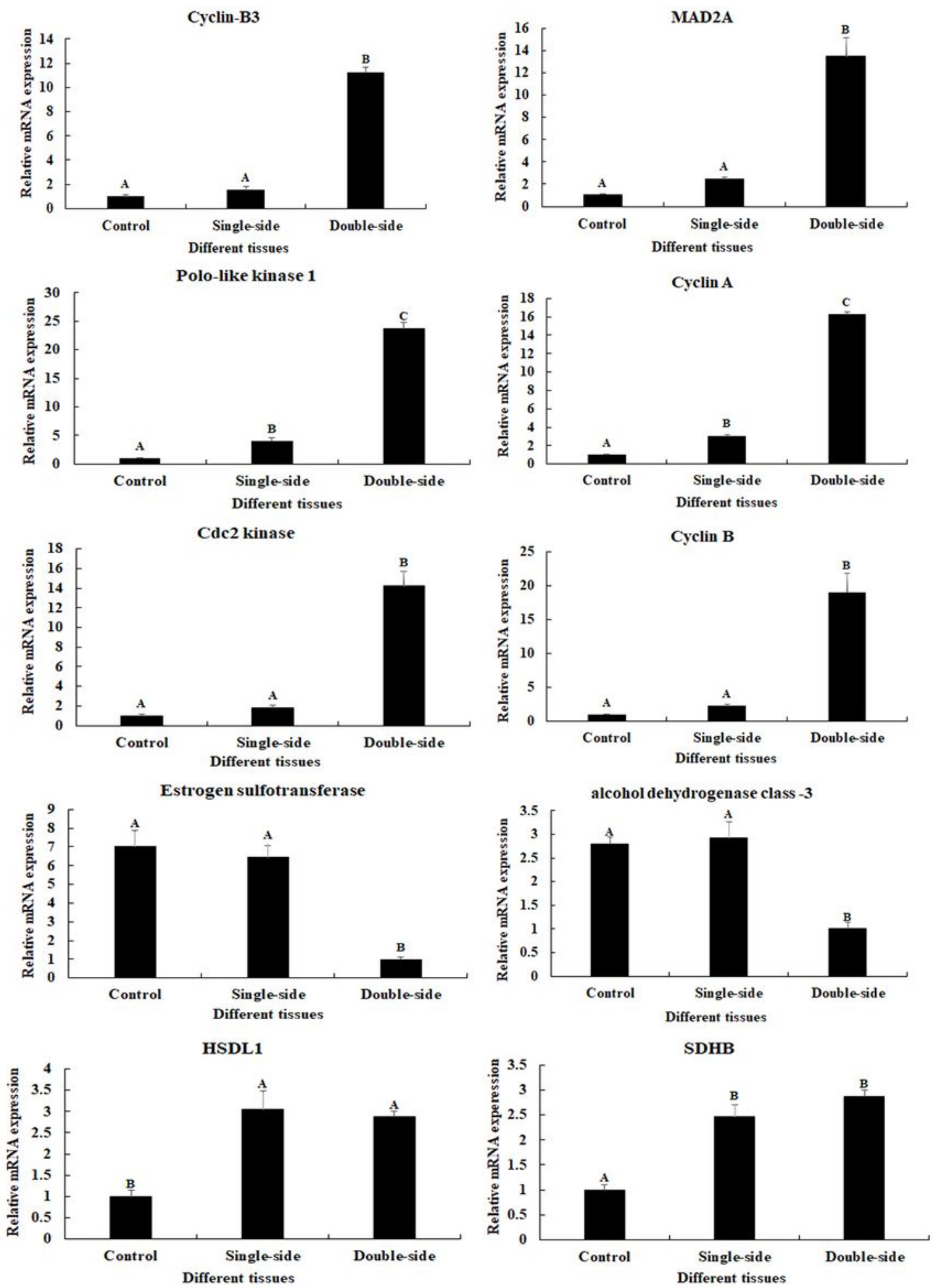

\section{Figure 4}

Verification of the expressions of 10 differentially expressed genes (DEGs) between the androgenic gland of normal prawns, prawns of single-side ablation and prawns of double-side ablation by qPCR. The amounts of DEGs expression were normalized to the EIF transcript level. Data are shown as mean \pm SD (standard deviation) of tissues in three separate individuals. Capital letter indicates expression. 


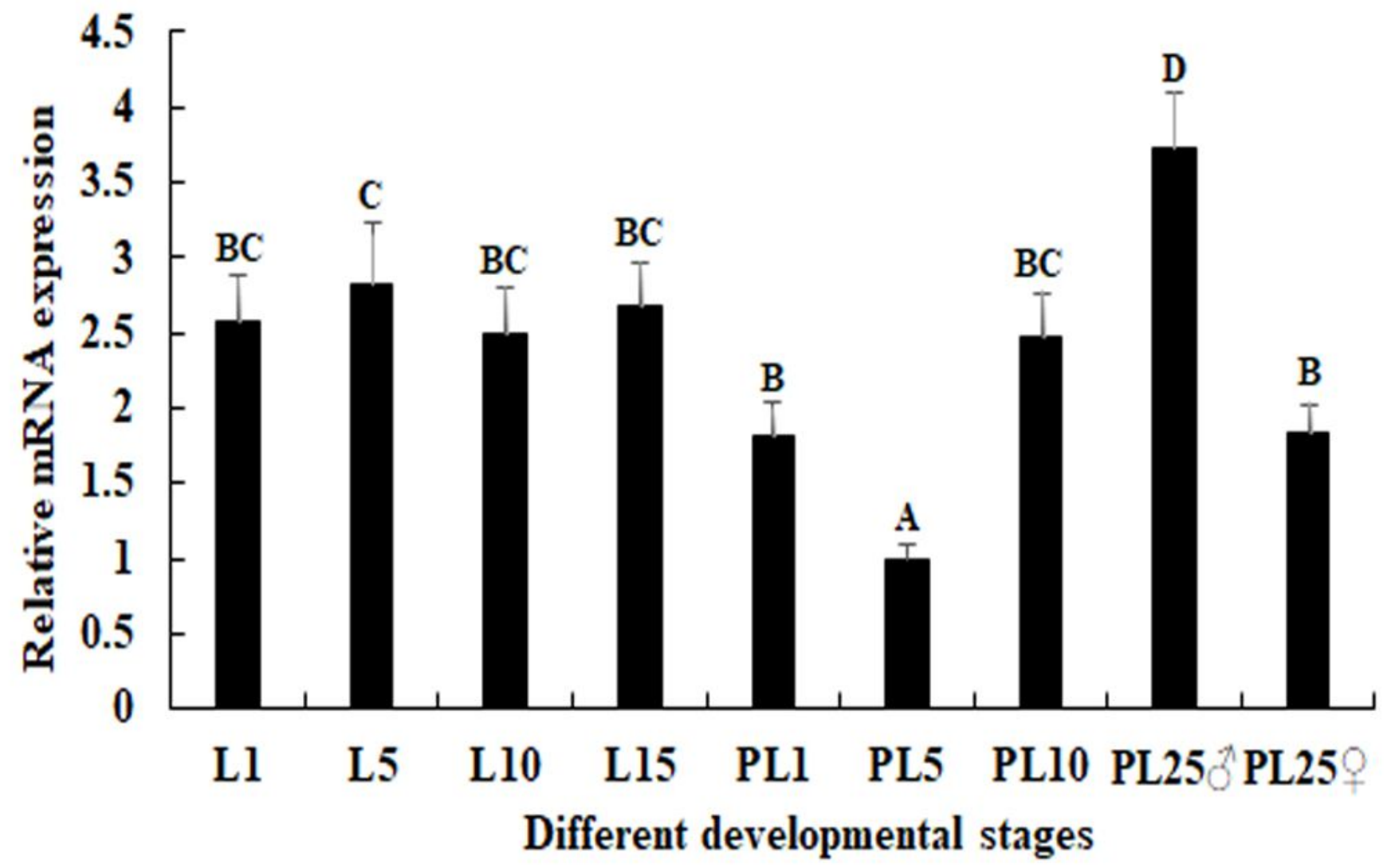

Figure 5

Expression characterization of Mn-HSDL1 in different developmental stages. The amount of Mn-HSDL1 mRNA was normalized to the EIF transcript level. Data are shown as mean +SD (standard deviation) of tissues from three separate individuals. Capital letters indicate expression difference between different samples. 

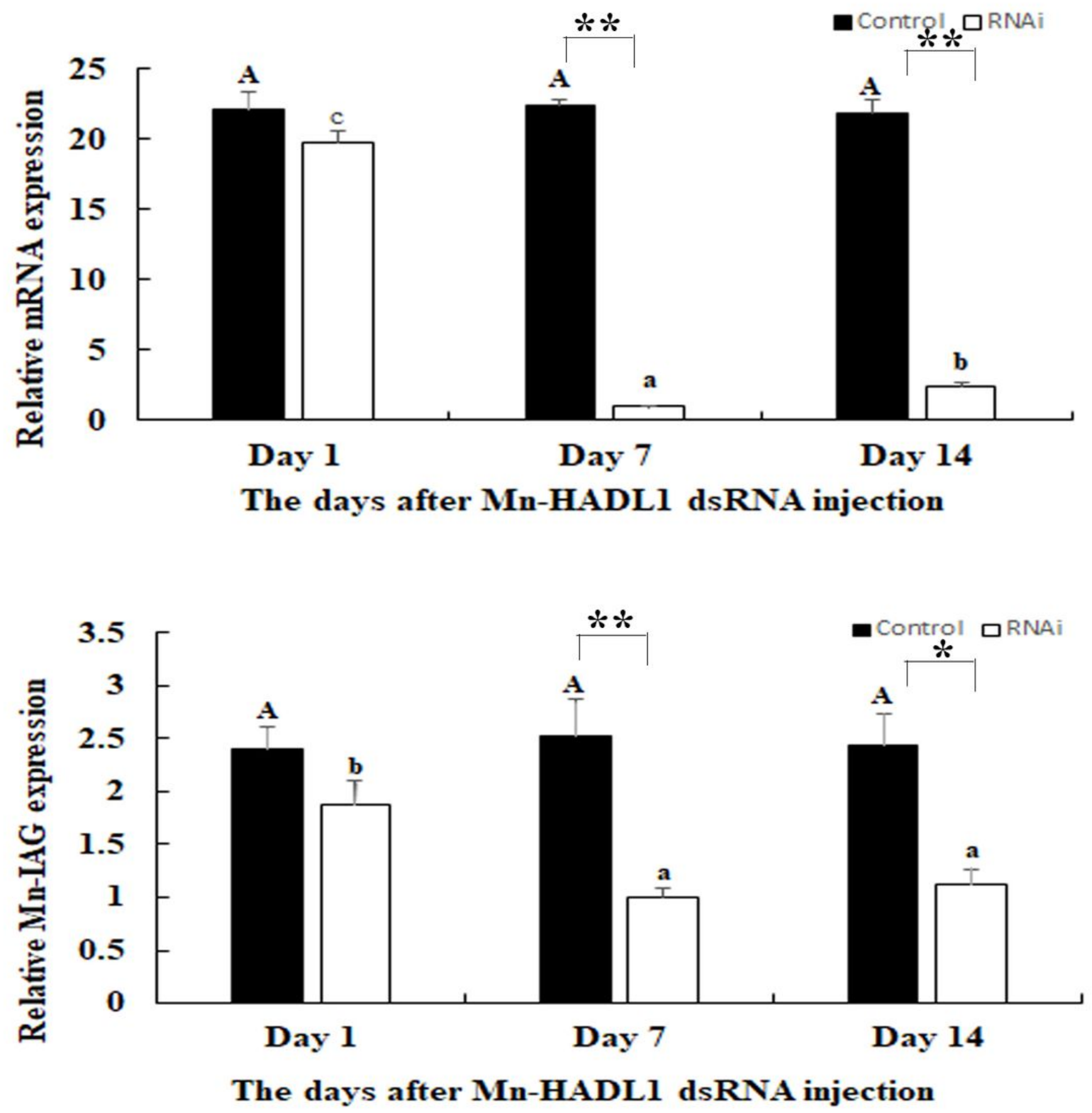

Figure 6

Expression characterization of Mn-HSDL1 and Mn-IAG at different days after Mn-HSDL1 dsRNA injection. The amount of Mn-HSDL1 and Mn-IAG mRNA was normalized to the EIF transcript level. Data are shown as mean +SD (standard deviation) of tissues from three separate individuals. Capital letters indicated expression difference between different days after GFP injection in control group. Lowercase indicated expression difference between different days after Mn-HSDL1 dsRNA injection in RNAi group. * $(p<0.05)$ and $* *(p<0.01)$ indicates significant expression difference between the RNAi group and control group at the sample day. A: Expression characterization of Mn-HSDL1 at different days after Mn-HSDL1 dsRNA injection. B: Expression characterization of Mn-IAG at different days after Mn-HSDL1 dsRNA injection. 

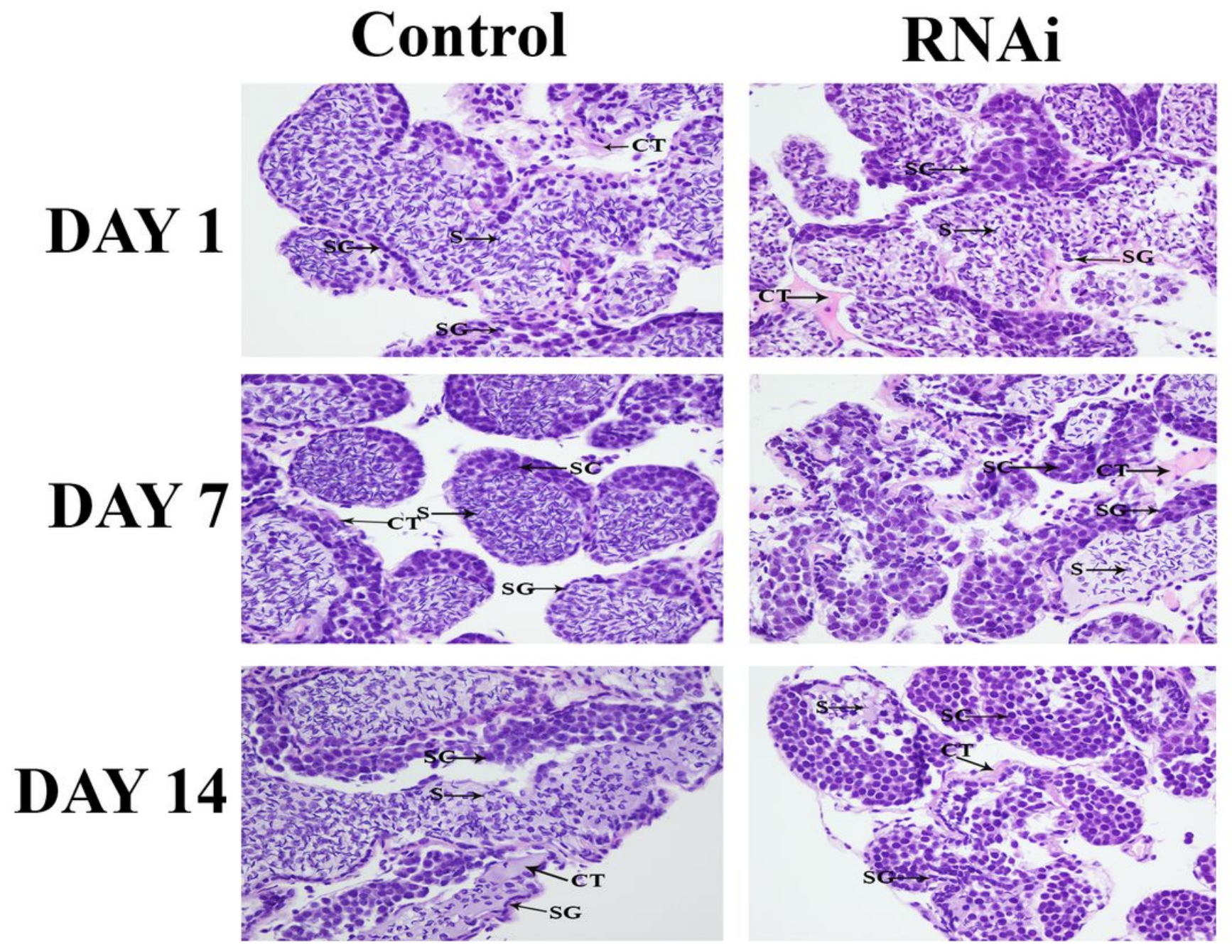

Figure 7

The histological observations of testis between RNAi and control group. SG: Spermatogonia; SC: spermatocyte; S: sperm; CT: collecting tissue. Scale bars $=20 \mu \mathrm{m}$.

\section{Supplementary Files}

This is a list of supplementary files associated with this preprint. Click to download.

- Controlday1.jpg

- RNAiday1.jpg

- Controlday14.jpg

- RNAiday14.jpg

- Controlday7.jpg

- RNAiday7.jpg 